\title{
ALGEBRAIC APPROACH TO SYMMETRIES AND INVARIANTS CONSTRUCTION
}

\author{
S. Andrianov*, SPbSU, St.-Petersburg, Russia
}

\begin{abstract}
This reports presents a new algebraic approach to approximating symmetries and invariants construction. Both invariants and symmetries are separated into dynamical and kinematical ones. Additionally each type of symmetries is separated into intrinsic and imposed symmetries. The intrinsic symmetries are generated by the dynamical system under study. The imposed symmetries ensure some desired properties of the dynamical system. This approach is very useful for optimal beam lines design problems. The kinematical invariants are used as a nonlinear theoretical probe. Such probes can be used for nonlinear effects investigation and control. Symmetries and invariants constructions procedure are based on the matrix formalism for the Lie algebraic methods. This formalism allows to create algebraic equations for determining block-matrices entering into the corresponding symmetries and invariants description. These equations can be solved in a symbolic mode, and the corresponding results are included in a special database. The algebraic approach is based on the Kronecker presentation of the Poincare-Witt basis for Lie algebras. All necessary statements are proved. Some practical applications for beam physics problems are discussed.
\end{abstract}

\section{INTRODUCTION}

It is known that such concept as emittance invariants has been used in particle beam physics for a long time. At first, this approach was developed for linear systems (the Courant-Snyder invariant, the time-dependent invariants by P.L.G.Leach, H.R.Lewis Jr.). In accelerator physics the problem of invariants plays the special role because of collective character of the object under study - particle beams. Besides this the problem is complicated by nonlinear aberrations which are inherent in any beamline. There are some works devoted to calculation of these important beam characteristics (see, e.g. [1]-[4]. In general case it is impossible to evaluate explicit invariants. The first problem discussed in this report is the problem of approximation invariants construction. The second is the problem of approximation symmetries searching. These coupling is explained their algebraic closeness. The knowledge of system symmetries is very important for design problems. They help to design the beamline with desired characteristics. The approach for retrieval of nonlinear approximation invariants is suggested in [1], [2]. But this approach is based on an infinite system of differential equations, which can be solved only numerically. A Lie-theoretical treatment of nonlinear beam dynamics has been given by Dragt [5] Here Lie the-

\footnotetext{
*serge@ apmath.spb.ru
}

ory is applied for investigation of the invariants-symmetry problems for beamlines using the matrix formalism for Lie algebraic methods [6].

\section{MATHEMATICAL BACKGROUNDS}

Dynamics of beam particles can be presented in the form of Lie nonlinear transformation $\mathcal{M}$ in the form of timeordered exponential operator

$$
\mathcal{M}\left(t \mid t_{0}\right)=\mathrm{T} \exp \left(\int_{t_{0}}^{t} \mathcal{L}_{\mathbf{F}(\tau)} d \tau\right),
$$

or the so called Magnus's presentation

$$
\mathcal{M}\left(t \mid t_{0}\right)=\exp \left(\mathcal{L}_{\mathbf{G}\left(t \mid t_{0}\right)}\right),
$$

where $\mathcal{L}_{\mathbf{F}(\tau)}$ is a Lie operator associated with some function $\mathbf{F}(\tau)=\mathbf{F}(\tau ; \mathbf{X}, \mathbf{U}, \mathbf{B})$, defining the motion equation for beam particles

$$
\frac{d \mathbf{X}}{d t}=\mathbf{F}(t ; \mathbf{X}, \mathbf{U}, \mathbf{B}),
$$

where $\mathbf{X} \in \mathcal{X}, \mathbf{U} \in \mathcal{U}, \mathbf{B} \in \mathcal{B}$ are a phase vector, a control functions vector and a control parameters vector respectively. The Eqs. (1), (2) define a dynamical system with control and the operator $\mathcal{M}$ can be identified with the dynamical system itself. The new function $\mathbf{G}\left(t \mid t_{0}\right)$ for the Lie operator $\mathcal{L}_{\mathrm{G}\left(t \mid t_{0}\right)}$ can be calculated using the continuous analogue of the well known CBH-formula [6].

\subsection{The Basic Definitions and Concepts}

Let give the following definitions:

D e f in ition 1 A set of transformations $\tau: \mathcal{T} \mapsto \hat{\mathcal{T}}$, $\mathcal{A}_{X}: \mathcal{X} \mapsto \hat{\mathcal{X}}, \mathcal{A}_{U}: \mathcal{U} \mapsto \hat{\mathcal{U}}, \mathcal{A}_{B}: \mathcal{B} \mapsto \hat{\mathcal{B}}$ is called a symmetry transformation of a dynamical system $\mathcal{M}$, if it guarantees the commutativity of the following diagram:

$$
\begin{aligned}
\mathcal{T} \times \mathcal{X} \times \mathcal{U} \times \mathcal{B} \stackrel{\mathcal{M}}{\longrightarrow} \mathcal{X} \\
\downarrow_{\tau \times \mathcal{A}_{X} \times \mathcal{A}_{U} \times \mathcal{A}_{B}} \downarrow_{\mathcal{A}} \\
\hat{\mathcal{T}} \times \hat{\mathcal{X}} \times \hat{\mathcal{U}} \times \hat{\mathcal{B}} \stackrel{\mathcal{M}}{\longrightarrow} \hat{\mathcal{X}}
\end{aligned}
$$

$\mathrm{D}$ e $\mathrm{f}$ i n i t i o 2 Let $\mathcal{A}$ be a symmetry transformation for the dynamical system $\mathcal{M}$ and $\mathcal{A}$ is a set of such transformations. Then a function $I(\mathbf{X}, t)$ is called a dynamical invariant, if $\mathcal{A} \circ I(\mathbf{X}, t)=I(\mathbf{X}, t)$, for some $\mathcal{A} \in \mathcal{A}$ and a kinematical invariants (compare with [3]), if $\mathcal{A} \circ I(\mathbf{X}, t)=$ $I(\mathbf{X}, t)$, i.e. $\mathcal{A} \circ I(\mathbf{X}, t)=I(\mathbf{X}, t) \forall \mathcal{A} \in \mathcal{A}$. 
D e f i n ition 3 Let a function $I(\mathbf{X}, t)$ be an invariant under symmetry transformations, then it is called the intrinsic dynamical invariant, if these transformations are generated by the dynamical system under study and the intrinsic kinematical invariants, if these transformations are generated by some class of dynamical systems.

For example, the authors of the work [3] introduced such kind of kinematical invariants for a class of Hamiltonian systems.

Let $\mathcal{L}_{\mathrm{G}}$ be a Lie operator associated with some vector function $\mathbf{G}(\mathbf{X}, t)$. This operator can be considered as an infinitesimal operator for transformation of some symmetry.

D e f i n i t i o n 4 If some function $\mathbf{F}(\mathbf{X}, t)$ defines the $d y$ namical system according to the Eq. 3 and the function $\mathbf{G}(\mathbf{X}, t)$ defines a transformation $\mathcal{N}=\exp \left(\mathcal{L}_{\mathbf{G}}\right)$, and there is the equality

$$
\left\{\mathcal{L}_{\mathrm{F}}, \mathcal{L}_{\mathrm{G}}\right\}=\mathcal{L}_{\mathrm{F}} \circ \mathcal{L}_{\mathrm{G}}-\mathcal{L}_{\mathrm{G}} \circ \mathcal{L}_{\mathrm{F}}=0
$$

then the transformation $\mathcal{N}=\exp \left(\mathcal{L}_{\mathbf{G}}\right)$ is called the symmetry transformation for the dynamical system (1)-(3).

It should be noted that instead of a single function $\mathbf{G}$ one can consider a set of functions $\mathcal{G}$ satisfying the Def. 4 .

\subsection{The Approximate Invariant and Symmetry}

In general case the retrieval of invariants and symmetries is very complicated problem. Taking into account that usually the Lie map is found using perturbation presentation we can redefine the conception of invariants and symmetry. The corresponding definitions can be rewritten by the following way. For an approximate symmetry one can formulate

D e finition 5 Let $\mathcal{L}_{\mathrm{F}}$ and $\mathcal{L}_{\mathrm{G}}$ be Lie operators for the dynamical system and the symmetry group correspondingly and takes place an equality $\left\{\mathcal{L}_{\mathbf{F}}, \mathcal{L}_{\mathbf{G}}\right\}=\mathcal{L}_{\mathbf{H}}$, where $\mathbf{H}(\mathbf{X}, t)=\sum_{k \geq 0} H_{k}(\mathbf{X}, t)$. Then, if $H_{k}(\mathbf{X}, t) \equiv 0 \forall k \leq N$ we shall tell that $\mathcal{L}_{\mathbf{G}}$ generates approximate symmetry of the $N$-th order.

and for an approximate invariant

Definition 6 Function $I^{(N)}(\mathbf{X}, t)$ is called an approximate invariant of the $N$-th order, if $\mathcal{L}_{\mathbf{G}} \circ I^{(N)}=$ $=\sum_{k \geq N+1} \mathrm{I}_{k}(\mathbf{X}, t)$, where $\mathrm{I}_{k}$ are scalar homogeneous polynomials of $k$-th order.

\section{THE MATRIX FORMALISM FOR THE PROBLEM}

\subsection{The Dynamic Invariants and Symmetries}

Let the functions $\mathbf{F}(\mathbf{X}, t)$ and $\mathbf{G}(\mathbf{X}, t)$ admit the following expansions $\mathbf{F}(\mathbf{X}, t)=\sum_{k=0}^{\infty} \mathbb{F}_{k}(t) \mathbf{X}^{[k]}$ and $\mathbf{G}(\mathbf{X}, t)=$ $\sum_{k=0}^{\infty} \mathbb{G}_{k}(t) \mathbf{X}^{[k]}$, where $\mathbf{X}^{[k]}$ is the Kronecker power of the $k$-th order for the phase vector $\mathbf{X}^{[k]}$ and $\mathbb{F}_{k}(t), \mathbb{G}_{k}(t)$ are two-dimensional matrices. Then using properties of the Kronecker product and sum one can evaluate the commutator from the Def. 5 and the following theorem can be proved.

The o r e m 1 The function generates a symmetry of the dynamical system (1)-(3) iff there are following equalities

$$
\begin{aligned}
& \mathbb{G}_{j} \mathbb{F}_{0}^{\oplus j}=\sum_{k=1}^{\infty}\left(\mathbb{F}_{k} \mathbb{G}_{j-k}^{\oplus k}-\mathbb{G}_{j-k} \mathbb{F}_{k}^{\oplus j-k}\right) \\
& 1 \leq k \leq j, \quad \forall j \geq 1 .
\end{aligned}
$$

Here $\mathbb{A}^{\oplus k}=\mathbb{A}^{\oplus(k-1)} \otimes \mathbb{E}+\mathbb{E}^{[k-1]} \otimes \mathbb{A}$ is the Kronecker $k$ multiple sum of some matrix $\mathbb{A}$. the set of the equalities (4) should be solved over the matrices $\mathbb{G}_{k}, k \geq 0$. In the case of an approximate symmetry of $N$-th order these equalities should be solved for all $j \leq N$.

For the construction of defining relations for approximate invariants we should write the following expansion for an invariant function: $I(\mathbf{X}, t)=\sum_{k=0}^{\infty} \mathbf{I}_{k}(t) \mathbf{X}^{[k]}$, where $\mathbf{I}_{k}(t)$ are vector functions be subject to definitions. According to the matrix formalism [6] the invariance condition can be written in the following differential form

$$
\frac{d \mathbf{I}_{k}(t)}{d t}+\sum_{j=1}^{k} \mathbb{P}^{j k}(t) \mathbf{I}_{k}(t)=0, \quad \forall k \geq 0,
$$

where $\mathbb{P}_{1 k}=\mathbb{F}_{k}$ (see above) and

$$
\mathbb{P}^{k j}=\mathbb{P}^{1(j-k+1)} \oplus \mathbb{P}^{(k-1)(j-1)}, j \geq k .
$$

The invariance condition can also be rewritten in the algebraic form:

$$
I\left(\mathbf{X}, t_{*}\right)=I\left(\mathcal{N}^{-1}\left(t_{*} \mid t\right) \circ \mathbf{X}, t\right)=I(\mathbf{I}, t),
$$

where $\mathcal{N}^{-1}\left(t_{*} \mid t\right)$ is an inverse operator for symmetry transformation $\mathcal{N}\left(t_{*} \mid t\right), t_{*}$ is some value of the independent variable $t, t_{*} \geq t$. The matrix presentation of all our objects leads to the following chain of matrix equations:

$$
\mathbf{I}_{k}(t)=\sum_{j=0}^{k}\left(\mathbb{T}^{j k}\left(t \mid t_{*}\right)\right)^{\mathrm{T}} \mathbf{I}_{j}\left(t_{*}\right)
$$

where $\mathbb{T}^{j k}\left(t \mid t_{*}\right)$ are expansion matrices for the inverse operator $\mathcal{N}^{-1}\left(t_{*} \mid t\right)$ :

$$
\mathcal{N}^{-1} \circ \mathbf{X}=\sum_{k=0}^{\infty} \mathbb{T}^{1 k}(t) \mathbf{X}^{[k]}
$$

and $\mathbb{T}^{k k}=\left(\mathbb{T}^{11}\right)^{[k]}, \mathbb{T}^{j k}=\sum_{\left(\begin{array}{c}i_{1}+\ldots+i_{j}=k \\ i_{l} \geq 1\end{array}\right)} \bigotimes_{l=1}^{j} \mathbb{T}_{1 i_{1}}, k \geq j$. It should be noted that matrices $\mathbb{T}^{1 k}$ can easily be calculated using the generalized Gauss's algorithm:

$$
\mathbb{T}^{11}=\left(\mathbb{N}^{11}\right)^{-1}, \quad \mathbb{T}^{1 k}=-\sum_{j=1}^{k} \mathbb{T}^{11} \mathbb{N}^{1 j}\left(\mathbb{T}^{11}\right)
$$


and

$$
\mathcal{N} \circ \mathbf{X}=\sum_{k=0}^{\infty} \mathbb{N}^{1 k}(t) \mathbf{X}^{[k]},
$$

\subsection{The Approximate Kinematical Invariants}

In the case of beam physics problems it is more preferable to use functions of moments $\mathbf{M}^{(k)}(t)=$ $\int_{\mathcal{M}(t)} f(\mathbf{X}, t) \mathbf{X}^{[k]} d \mathbf{X}$ instead of functions $I(\mathbf{X}, t)$. Here $\mathcal{M}(t)$ is an image of an initial phase set $\mathcal{M}_{0}$ and $f(\mathbf{X}, t)$ is a distribution function in the phase space for some $t$. From the definition of moments one can write

$$
\mathbf{M}^{(k)}(t)=\int_{\mathcal{M}_{0}} f_{0}\left(\mathcal{N}^{-1} \circ \mathbf{X}^{[k]}\right) \mathrm{J}(\mathcal{N}) d \mathbf{X},
$$

where $J(\mathcal{N})$ is the Jacobian for the transformation $\mathcal{N}$. For the conservative Hamiltonian systems we have $\mathrm{J}(\mathcal{N}) \equiv 1$ and in this case we can be rewritten (using the matrix formalism):

$$
\mathbf{M}^{(k)}(t)=\sum_{j=k}^{\infty} \mathbb{T}^{j k}\left(t \mid t_{0}\right) \mathbf{M}^{(j)}\left(t_{0}\right),
$$

It should be noted that the problem of kinematical invariants and symmetries is solved differently. Here we shall consider an example of kinematical invariants. The construction method for such kind of invariants for Hamiltonian systems was suggested in the work [3]. In general, for this purpose we use the tool of Kronecker matrix operators and Casimir operators for corresponding Lie algebra. In particular, for kinematical invariants of linear symplectic dynamical systems we have

$$
\begin{aligned}
& I_{2 k}(\mathbf{M})=(-1)^{k}\left(\mathbf{M}_{2}^{[k]}\right)^{\mathrm{T}} \mathbb{J}^{\{k\}} \mathbf{M}_{2}^{[k]}= \\
& \quad=(-1)^{k} \operatorname{Sp}\left(\mathbb{M}_{2}^{\{k\}} \mathbb{J}^{\{k\}}\right)=(-1)^{k} \operatorname{Sp}\left(\left(\mathbb{M}_{2} \mathbb{J}\right)^{k}\right),
\end{aligned}
$$

where $\mathbf{M}_{2}=\left\langle\mathbf{X}^{[2]}\right\rangle=\int_{\mathcal{M}} f(\mathbf{X}) \mathbf{X}^{[2]} d \mathbf{X}$ and $\mathbf{M}_{2}^{[k]}=\mathbf{M}_{2} \otimes$ $\ldots \otimes \mathbf{M}_{2},\{k\}$ denotes the symmetrized Kronecker power of the $k$-th order, $\mathbb{M}_{2}=\mathbf{M}_{2} \mathbf{M}_{2}^{\mathrm{T}}$ is a matrix of second moments.

For nonlinear dynamical systems the appropriate kinematical invariants of $2 N$-th order $-J_{2 N}$ is constructed in the form (compare with [4])

$$
J_{2 N}=\sum_{l=1}^{N} \alpha_{l}^{(2 N)} I_{2 l} J_{2(N-l)}, \quad J_{0}=1,
$$

where $\alpha_{l}^{(2 N)}$ are some constants which should be worked out, $I_{2 l}, l<N$ - kinematical invariants of corresponding orders for linearized system. Note that $I_{(2 l)}$ depends on a distribution function $f(\mathbf{X})$ (its centrality leads to equalities $I_{(2 l+1)}=0$ ), therefore parameters defining distribution functions affects values of $J_{2 N}$ too. Using methods of differential geometry one can approximate current phase space set $\mathcal{M}(t)$ by some parameters (e.g. such parameters as concavity degree, twisting degree and so on) in the class of polynomials over these parameters. It is possible to suggest the three steps algorithm of nonlinear invariants construction:

Step 0. An initial distribution function is described as the function of some parameters. Polynomial approximations for linear kinematical invariants $I_{2 j} j \leq N$ are calculated as function both of distribution function parameters and beamline parameters. Here it is possible to present the distribution function as a polynomial function of geometric and topological properties of the current distribution function.

Step 1. A set of formal nonlinear invariants is determined according to the Eq. (5). As a result the desired nonlinear invariants $J_{2 N}$ is the polynomial of $2 N$-th order with respect to these parameters.

Step 2. One should build a combination of the kinematical invariants for linearized system $I_{2 j}, j \leq N$. This combination should not depend on dynamical parameters up to some order.

The described approach gives a flexible and effective tool for investigations of nonlinear aberrations. The most part of necessary computing operations can be evaluated using computer algebra codes on some general assumptions. Then this prepared information is used for numerical calculations. As a result one can construct a probe for testing nonlinear effects of different nature. We should note that this probe is built on the measured physical variables because the linear invariants are functions of partial emittances $\varepsilon_{x}^{2}, \varepsilon_{y}^{2}, \ldots$.

\section{REFERENCES}

[1] Antillon A., Hoeneisen B. Emittance of a Nonlinear Machine: the Two-dimensional Problem, Nucl. Instr. and Meth. in Physics Res., A305, No 2, 239-246 (1992).

[2] Antillon A., Forest E., Hoeneisen B., Leyvraz F. Transport Matrices for Nonlinear Lattice Functions, Nucl. Instr. and Meth. in Physics Res., A305, No 2, 247-256 (1992).

[3] Dragt A.J., Gluckstern R.L., Neri F., Rangarajan G. Theory of Emittance Invariants, Lectures Notes in Physics, 343 Froniers of Particle Beams Observation, Diagnosis and Correction /Eds. M.Month, S.Turner/. Berlin: Springer, 94-121 (1989).

[4] Lysenko W.P., Oversley M.S. Moment Invariants for Particle beams. Linear Accelerator and Beam Optics Codes /Ed. Eminhizer C.R./ AIP Conf. Proc., No 177, 323-335 (1988).

[5] Dragt A.J. Lectures on Nonlinear Orbit Dynamics, Physics of High Energy Particle Accelerators. (Eds. Carrigan, F.R.Huson, M.Month M.) AIP Conf. Proc., No 87. 147-313 (1987).

[6] Andrianov S.N. A Matrix Representation of the Lie Algebraic Methods for Design of Nonlinear Beam Lines. Proc. of the 1996 Comp. Accel. Physics Conf., Williamsburg, Virginia, USA /Eds. J.J.Bisognano, A.A.Mondelli/. AIP Conf. Proc., No 391. 355-360 (1997). 